\title{
Trans-Cinnamaldehyde Increases Random Pattern Flap Survival Through Activation of the Nitric Oxide Pathway
}

This article was published in the following Dove Press journal: Drug Design, Development and Therapy

\author{
Xiaobin Luo' \\ Bin Zhao ${ }^{2}$ \\ Baoxia Chen ${ }^{2}$ \\ Hongyu Chen' \\ Tao Han' \\ Najeeb Bassam Najeeb Bsoul' \\ Hede Yan (D) \\ 'Department of Orthopedics (Division of \\ Hand Surgery), The Second Affiliated \\ Hospital and Yuying Children's Hospital \\ of Wenzhou Medical University, Key \\ Laboratory of Orthopedics of Zhejiang \\ Province, The Second School of \\ Medicine, Wenzhou Medical University, \\ Wenzhou, Zhejiang, 325027, People's \\ Republic of China; ${ }^{2}$ Department of Post \\ Anaesthesia Care Unit, The Second \\ Affiliated Hospital and Yuying Children's \\ Hospital of Wenzhou Medical University, \\ The Second School of Medicine, \\ Wenzhou Medical University, Wenzhou, \\ 325027, Zhejiang, People's Republic of \\ China
}

Correspondence: Hede Yan

Department of Orthopaedics (Division of Hand Surgery), The Second Affiliated Hospital and Yuying Children's Hospital of Wenzhou Medical University, West

Xueyuan Road 109\#, Wenzhou, 325027,

Zhejiang Province, People's Republic of

China

Email yanhede@hotmail.com
Background: The application of random pattern skin flaps is limited in plastic surgery reconstruction due to necrosis. Trans-cinnamaldehyde has antibacterial, anticancer, and antioxidant properties. In this study, we aimed to investigate the effect of transcinnamaldehyde on skin flap survival and its possible mechanism regarding nitric oxide.

Materials and Methods: One hundred forty male Sprague-Dawley rats were randomly divided into seven groups $(\mathrm{n}=20$ each group). After the dorsal flap was raised, different doses of trans-cinnamaldehyde $(10,20$, and $30 \mathrm{mg} / \mathrm{kg})$ were immediately given by oral gavage in the three different groups. To assess the possible involvement of the nitric oxide system, $\mathrm{N}^{\mathrm{G}}$-nitro-L-arginine methyl ester (L-NAME, a nonselective nitric oxide synthase inhibitor) was used in this study. All flap samples were incised on postoperative day 7 .

Results: Our results showed that flap survival was increased significantly in the $20 \mathrm{mg} / \mathrm{kg}$ (P $<0.001$ ) trans-cinnamaldehyde (TC) group compared to the control group or $30 \mathrm{mg} / \mathrm{kg} \mathrm{TC}$ group. This protective function was restrained by coadministration of L-NAME with $20 \mathrm{mg}$ / $\mathrm{kg}$ TC. The results of histopathology, laser Doppler, arteriography mediated with oxidegelatine, and fluorescent staining all showed a significant increase in capillary count, collagen deposition, angiogenesis, and flap perfusion. Immunohistochemistry results revealed a significant increase in the expression of CD34, eNOS, and VEGF.

Conclusion: Trans-cinnamaldehyde increased flap survival through the nitric oxide synthase pathway and contributed to angiogenesis. A concentration of $20 \mathrm{mg} / \mathrm{kg}$ transcinnamaldehyde was recommended in this study.

Keywords: random pattern flap, flap necrosis, trans-cinnamaldehyde, angiogenesis, flap perfusion

\section{Introduction}

Since the mid-1960s, random pattern flaps have been used in injuries, tumour excision, and malformations. ${ }^{1}$ Random pattern flaps have been designed without any regard to any known vessels other than the subdermal plexus. However, unstable flap survival, such as distal portion necrosis, limits the use of random pattern flaps ${ }^{2}$ even though microsurgical techniques for flap transplantation have been further improved and developed. Based on the angiosome theory, ${ }^{3}$ the blood supply of the skin flap is supported by the main supplier artery. Therefore, perforator arteries are cut when the flap is elevated, and skin perfusion is completely supported by dermal superficial and deep arterial plexuses. 
Various ways of increasing flap survival have been used, such as surgical delay, venous superdrainage, and artery supercharge techniques, as well as vascular endothelium growth factors. ${ }^{4-7}$ However, these methods are expensive and extend the operative time. Therefore, selecting a suitable pharmacologic is a great alternative therapy to improve skin flap survival. Many different pharmacologic therapies, such as antithrombosis and anti-inflammation, have been reported; however, these therapies are not reliable in clinical practice. ${ }^{8-10}$ There are various factors affecting arterial supply. GarciaPedraza et al, ${ }^{11}$ reported that vasodilation factors, such as prostacyclin and nitric oxide, as well as contracting factors, such as thromboxane A2 and endothelin-1, have important influences on flap survival.

Trans-cinnamaldehyde (TC; C9H8O, Figure 1) is one of three major components of cinnamon oil isolated from Cinnamomum zeylanicum, and the other two components are eugenol and linalool. ${ }^{12}$ In general, cinnamon is considered a remedy due to its active component, TC. Previous studies have reported that TC has antibacterial, anticancer, and antioxidant properties. ${ }^{12,13} \mathrm{Kim}$ et al, ${ }^{14}$ suggested that $\mathrm{TC}$ has beneficial anti-inflammatory effects on endothelial cells and macrophages in vitro. Subash Babu et al, ${ }^{15}$ revealed that oral administration of cinnamaldehyde $(20 \mathrm{mg} / \mathrm{kg}$ bodyweight $)$ significantly decreases glycosylated haemoglobin (HbAlc), serum total cholesterol, and triglyceride levels. In addition, Ayano et $\mathrm{al}^{16}{ }^{16}$ reported that cinnamaldehyde induces relaxation of rat aortae and that the vasodilation effect

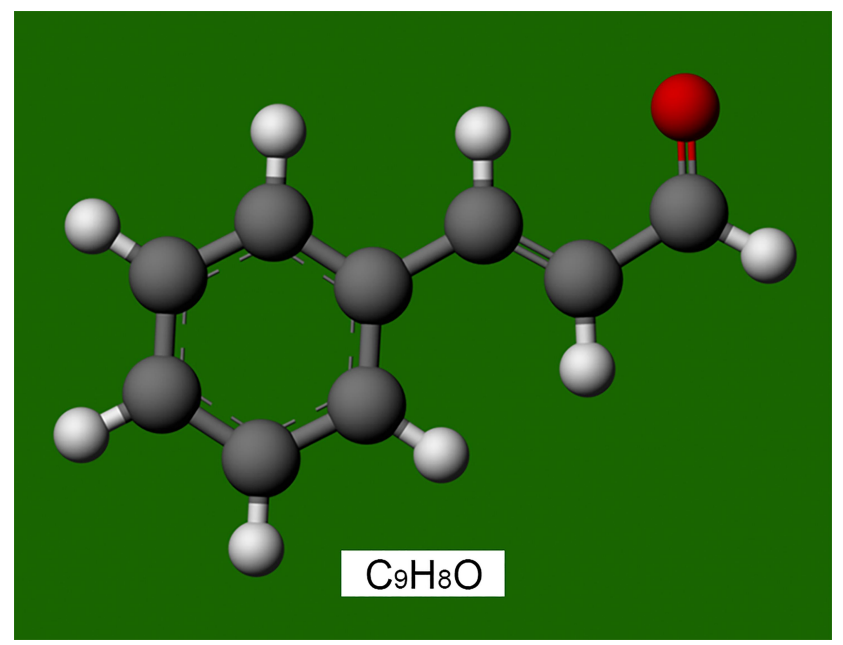

Figure I Chemical mode structure of trans-cinnamaldehyde $(\mathrm{C} 9 \mathrm{H} 8 \mathrm{O})$. The red dot represents the oxygen atom. is inhibited by $\mathrm{N}^{\mathrm{G}}$-nitro-L-arginine methyl ester (L-NAME). This vasodilation effect results from nitric oxide-mediated endothelial relaxation and smooth muscle hyperpolarization. ${ }^{17}$ Therefore, we speculated that trans-cinnamaldehyde may also have a vasodilation effect on capillaries through the potential participation of the nitric oxide system. Chin et al, ${ }^{18}$ demonstrated that the involvement of nitric oxide synthase is associated with angiogenesis in wound repair. Therefore, in this study, we investigated the effect of trans-cinnamaldehyde on flap survival in rats and the role of the nitric oxide pathway.

\section{Materials and Methods Ethics Statement}

All procedures received the animal care committee's approval of Wenzhou Medical University (approval code: wydw2019-0954). The conditions of the housing facilities, the care of laboratory animals and the animal experimental procedures were consistent with the national standard "Laboratory Animal-Guideline for Ethical Review of Animal Welfare" (GB/T35892-2018) of China.

\section{Animals and Models}

One hundred forty Sprague-Dawley rats (weighing $220 \pm 20$ g) were obtained from the animal house of Wenzhou Medical University. Rats were anaesthetized before surgical procedures through intraperitoneal injection of $1 \%$ sodium pentobarbital $(1.0 \mathrm{~mL} / \mathrm{kg})$. All rats were classified into seven groups, and the group classification is shown in Table 1. A random flap was generated by making an incision in the skin along an indicated line $(2 \mathrm{~cm} \times 8 \mathrm{~cm})$. To ensure that the flap perfusion was supported only through the caudal pedicle, all named perforator arteries beneath the flap, including bilateral deep circumflex iliac vessels (indicated by the two red circles in Figure $2 \mathrm{~A}$ ), were cut before elevating the bipedicled flap (indicated by the two black arrows in Figure 2B). Next, the flap was sutured back in place using 4-0 sutures interruptedly, and the sterile dressing was applied to the wound area.

\section{Drugs}

TC with a purity $\geq 95 \%$ (YZ-6023) and dimethyl sulfoxide (D8370) were purchased from Solarbio (CN). Purity $\geq 98 \%$ of L-NAME (N5751) was purchased from Sigma (US). L-NAME was dissolved in physiologic normal saline, and TC was dissolved in $0.5 \%$ DMSO.${ }^{15}$ L-NAME was used at a concentration of $10 \mathrm{mg} / \mathrm{kg}$. All drugs were kept in a $4^{\circ} \mathrm{C}$ refrigerator and administered freshly. 
Table I Treatment Groups, Average Flap Survival and Perfusion

\begin{tabular}{|l|l|l|l|}
\hline Groups & Treatment Identification & Flap Survival Rates (Mean \pm SD\%) & Flap Perfusion (PU \pm SD) \\
\hline 1 & Control (0.9\% normal saline) & $31 \pm 8$ & $255 \pm 20$ \\
2 & $10 \mathrm{mg} / \mathrm{kg}$ of TC & $47 \pm 7$ & $350 \pm 17$ \\
3 & $20 \mathrm{mg} / \mathrm{kg}$ of TC & $73 \pm \mathrm{II}$ & $402 \pm 25$ \\
4 & $30 \mathrm{mg} / \mathrm{kg}$ of TC & $33 \pm 6$ & $264 \pm 28$ \\
5 & $20 \mathrm{mg} / \mathrm{kg}$ of TC + L-NAME & $31 \pm 9$ & $243 \pm 15$ \\
6 & I mL/kg DMSO (0.5\%) & $28 \pm 6$ & $234 \pm 14$ \\
7 & L-NAME & $31 \pm 4$ & $244 \pm 23$ \\
\hline
\end{tabular}

Abbreviations: TC, trans-cinnamaldehyde; L-NAME, $\mathrm{N}^{\mathrm{G}}$-nitro-L-arginine methyl ester; DMSO, dimethylsulfoxide.

\section{Study Design}

Different doses of TC on flap survival were investigated to assess the dose response profile of the drug. TC was administered by oral gavage at concentrations of 10, 20, and $30 \mathrm{mg} / \mathrm{kg}$ immediately before elevating the flap. Moreover, the effective dose of $10 \mathrm{mg} / \mathrm{kg}$ of a nonselective nitric oxide synthase inhibitor (L-NAME) was co-administered intraperitoneally with the effective dose of $\mathrm{TC}$ to illustrate the possible influence of the nitric oxide pathway in this treatment. One hundred forty male Sprague-Dawley rats were assigned to the following groups: 42 rats were used for fluorescein staining in seven groups ( $\mathrm{n}=6$ each group); 42 rats were used for microvascular examination in seven groups using arteriography with oxide-gelatine contrast medium ( $\mathrm{n}=6$ each group); and 56 rats were used for histopathology and immunohistochemistry evaluation in seven groups ( $\mathrm{n}=8$ each group).

\section{Fluorescein Staining}

A dose of $5 \%$ fluorescein solution $(125 \mathrm{mg} / \mathrm{kg}$; Sigma) was injected on postoperative day 7 through the caudal vein in all groups ( $n=6$ in each group) to evaluate the percentage of vascular perfusion. ${ }^{19}$ Fluorescent staining images were distinct under ultraviolet light and recorded within 10 minutes.

\section{Laser Doppler Perfusion}

Laser Doppler perfusion images of the flap field $(2 \mathrm{~cm}$ $\mathrm{X} 8 \mathrm{~cm}$ ) were obtained (Moor, Axminster, UK) at $25^{\circ} \mathrm{C}$ when rats were anaesthetized. Blood perfusion of the flaps ( $n=8$ in each group) was detected on postoperative day 7 . The mean value was used.

\section{Microvascular Examination}

To evaluate the microvasculature density on postoperative day $7,{ }^{20}$ rats ( $\mathrm{n}=6$ in each group) were randomly selected for arteriography with oxide-gelatine contrast medium injected through the common carotid artery. After the arteriography, the rat bodies were kept at $4{ }^{\circ} \mathrm{C}$ overnight for fixation. The flaps were incised and radiographed using an X-ray machine (KUBTEC Xpert80, USA). The mean capillary density was calculated using vessel $\mathrm{J}^{21}$ a plugin of ImageJ.

\section{Histopathology}

3 Samples $(1 \mathrm{X} 1 \mathrm{~cm})$ in each rat were randomly harvested on day 7 postoperatively and incubated in $4 \%$ paraformaldehyde for 48 hours. Samples were then embedded in paraffin and cut into 5- $\mu \mathrm{m}$ sections. Each haematoxylin and eosin (H\&E)-stained slice was randomly observed for capillary count in 5 different areas $(100 \times$ magnification). The mean value was used. Masson's trichrome staining was performed to evaluate fibrin deposition in each sample, and 5 different areas of the sections were randomly measured $(100 \times$ magnification). The mean value was used.

\section{Immunohistochemistry (IHC)}

On postoperative day 7, 3 Samples $(1 \mathrm{X} 1 \mathrm{~cm})$ in each rat were randomly harvested and were embedded in paraffin, dehydrated with alcohol, and cut into 5- $\mu \mathrm{m}$ sections. Xylene and alcohol were used for section deparaffinization. Subsequently, the sections were incubated in 3\% $\mathrm{H}_{2} \mathrm{O}_{2}$ for 30 minutes. Bovine serum albumin (3\%) was used to block the nonspecific sites for 30 minutes. The sections were then incubated with mixtures of PBS and primary antibodies against CD34 (1:200, ab81289, Abcam), VEGF (1:200, ab231260, Abcam) and eNOS (1:200, ab76198, Abcam) overnight at $4^{\circ} \mathrm{C}$. A goat antibody labelled with horseradish peroxidase was used as the secondary antibody. Finally, all sections were counterstained using haematoxylin for 5 minutes. Images of sections were observed using a LSM 800 image acquisition system (Zeiss, Germany) and assessed using the IHC Tool Box, ${ }^{22}$ a plugin of ImageJ. 


\section{Statistical Analyses}

The results are presented as the mean \pm SD. Flap survival and pathologic studies were calculated and presented using one-way analysis of variance (ANOVA) followed by Tukey's test. SPSS Version 22.0 software was used for statistical analysis. $\mathrm{P}<0.05$ was considered statistically significant.

\section{Results}

\section{Viability Assessment}

On postoperative day 7 , the macroscopic survival areas of the flaps in the seven groups were marked with red curved lines (Figure $2 \mathrm{C}-\mathrm{I}$ ) by two investigators who were blinded to the group classifications. The statistical results are shown in Table 1. The flap survival area in the $20 \mathrm{mg} / \mathrm{kg}$ TC group

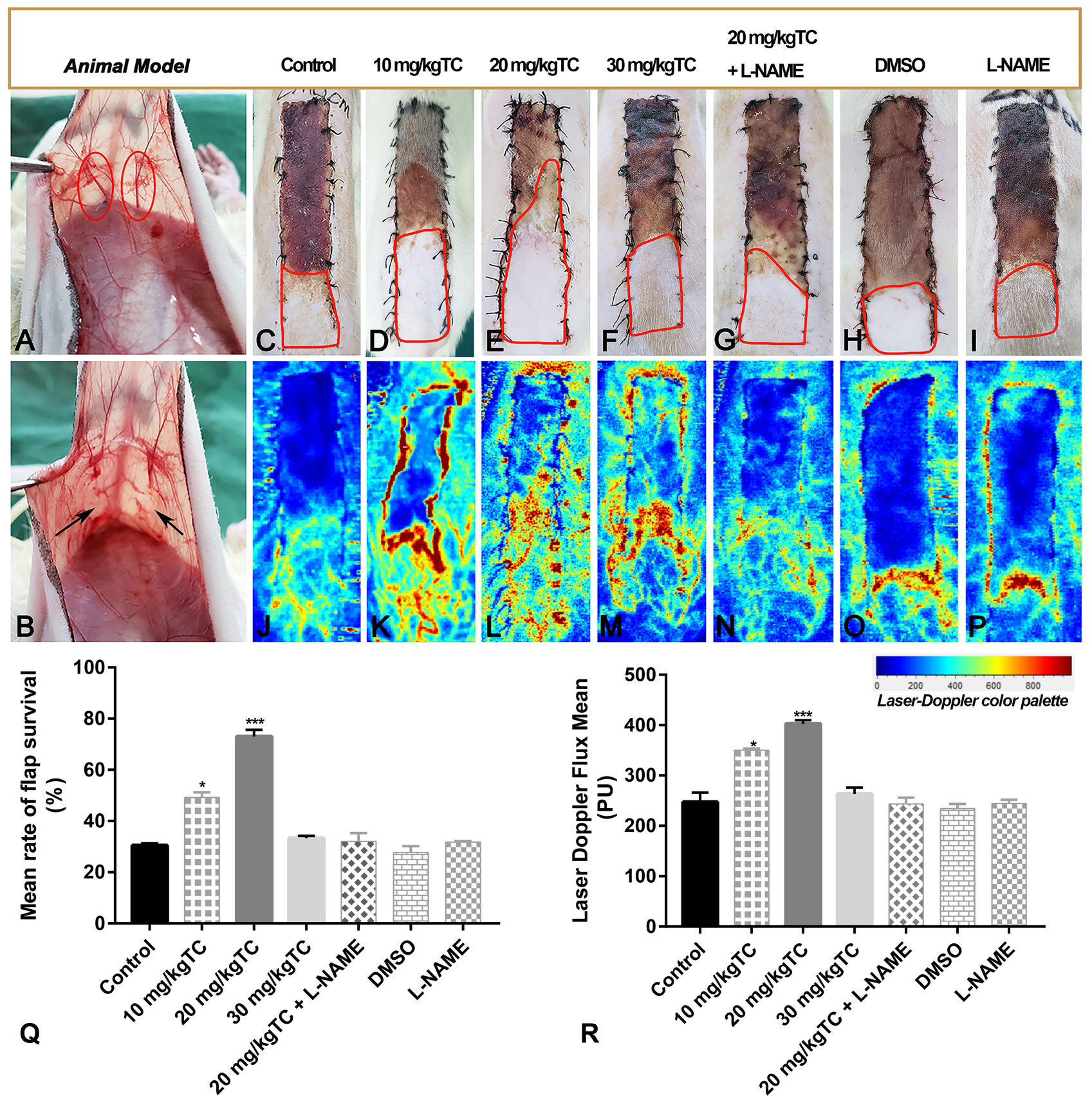

Figure 2 Intraoperative animal model and postoperative flap survival.

Notes: (A) Bilateral deep circumflex iliac vessels are indicated by two red circles. (B) Bilateral deep circumflex iliac vessels were cut before elevating the bipedicled flap, which are indicated by two black arrows. (C-I) On postoperative day 7, the macroscopic survival areas of flaps in the seven groups are marked with red curved lines. (J-P) The flap perfusion of the seven groups was detected using a laser Doppler system. (Q) Statistical percentage of flap survival. (R) Statistical results of flap perfusion. Significant differences between groups are indicated as $* * * p<0.001$, ${ }^{*} \mathrm{p}<0.05$. 
was the highest compared with the other six groups (Figure $2 \mathrm{Q} ; * * * \mathrm{P}<0.001)$. Flap survival in the $10 \mathrm{mg} / \mathrm{kg}$ TC group was higher than that in any of the control groups receiving normal saline, L-NAME, and DMSO. (*P < 0.05). There was no significant difference among the $30 \mathrm{mg} / \mathrm{kg} \mathrm{TC}$, $20 \mathrm{mg} / \mathrm{kg}$ TC + L-NAME, and control groups ( $\mathrm{P}>0.05)$ These significant differences were consistent with the results of laser Doppler. On postoperative day 7, laser Doppler perfusion imaging (Figure 2J-P) revealed that blood perfusion in the $20 \mathrm{mg} / \mathrm{kg}$ TC group was the highest among the seven groups (Figure 2R; ***P $<0.001$ ), and the mean blood perfusion in the seven groups is shown in Table 1. Blood perfusion in the $10 \mathrm{mg} / \mathrm{kg}$ TC group was higher than that in any of the control groups receiving normal saline, L-NAME, and DMSO. ( $\left.{ }^{*} \mathrm{P}<0.05\right)$. There was no significant difference among the $30 \mathrm{mg} / \mathrm{kg}$ TC, $20 \mathrm{mg} / \mathrm{kg} \mathrm{TC}+$ L-NAME, and control groups $(\mathrm{P}>0.05)$.

\section{Area and Concentration of Neovascular (CNV)}

On postoperative day 7, neovasculature was formed, and fluorescent staining revealed the area of vascular perfusion where flaps were filled (Figure $3 \mathrm{~A}-\mathrm{G}$ ). The mean percentage of flap-positive fluorescent staining in the seven groups is shown in Table 2. The percentage of fluorescent staining in the $20 \mathrm{mg} / \mathrm{kg}$ TC group was the highest among all groups (Figure $3 \mathrm{H} ; * * * \mathrm{P}<0.001$ ). The percentage of fluorescent staining in the $10 \mathrm{mg} / \mathrm{kg}$ TC group was higher than that in any of the control groups receiving normal saline, L-NAME, and DMSO. ( $* \mathrm{P}<0.05)$, but there was no significant difference between the control group and the $30 \mathrm{mg} / \mathrm{kg}$ TC group (P > $0.05)$. In addition, we verified the concentration of neovasculature $(\mathrm{CNV})$ through arteriography with oxide-gelatine contrast medium (Figure $3 \mathrm{I}-\mathrm{O}$ ). ${ }^{20}$ On postoperative day 7 , the mean percentage of microvessels in all groups is shown in Table 2. Significant differences in the density of neovasculature within the flap are shown in Figure 3P.

\section{Histopathological Studies}

On postoperative day 7, H\&E staining (Figure 4A-E, Left) and Masson's trichrome staining (Figure 4A-E, Right) were performed to determine capillary count and fibrin deposition, respectively. The $\mathrm{H} \& \mathrm{E}$ results showed that the number of capillaries in the $20 \mathrm{mg} / \mathrm{kg}$ TC group was the highest among the five groups (***P $<0.001)$, and the number of capillaries in the $10 \mathrm{mg} / \mathrm{kg} \mathrm{TC}$ group was greater than that in the control group (Figure $4 \mathrm{~F} ; * * * \mathrm{P}<$
0.001). There was no significant difference among the control group, the $20 \mathrm{mg} / \mathrm{kg} \mathrm{TC}+\mathrm{L}-\mathrm{NAME}$ group and the L-NAME group ( $p>0.05$ ). The results of Masson's trichrome staining suggested that the level of fibrin deposition in the $20 \mathrm{mg} / \mathrm{kg}$ TC group was the highest among the five groups and that the level of dermofibrosis in the $10 \mathrm{mg} / \mathrm{kg}$ TC group was higher than that in the control group (Figure $4 \mathrm{G} ; * * * \mathrm{P}<0.001$ ). There was no significant difference among the control group, the $20 \mathrm{mg} / \mathrm{kg} \mathrm{TC}+$ L-NAME group and the L-NAME group ( $\mathrm{p}>0.05)$.

\section{Expression Level of CD34, eNOS, and VEGF}

On postoperative day 7 , the immunohistochemistry results (Figure 5A-C) revealed that the expression levels of CD34, eNOS, and VEGF were the highest in the $20 \mathrm{mg}$ / $\mathrm{kg}$ TC group $(* * * \mathrm{p}<0.001)$, and the expression levels of these proteins in the $10 \mathrm{mg} / \mathrm{kg} \mathrm{TC}$ group were between those of the control group and the $20 \mathrm{mg} / \mathrm{kg}$ TC group with significant differences $(* * * p<0.001)$. However, there were no significant difference among the control group, $20 \mathrm{mg} / \mathrm{kg}$ TC+L-NAME and L-NAME ( $\mathrm{p}>0.05)$. Significant differences in the expression of CD34, eNOS, and VEGF are shown in Figure 5D-F.

\section{Discussion}

The present study demonstrated that trans-cinnamaldehyde (TC) improved skin flap survival through the nitric oxide pathway. In addition, we found that the most effective dose of TC was $20 \mathrm{mg} / \mathrm{kg}$ because the percentage of flap survival and the quantity of blood perfusion did not improve significantly with the application of lower or higher concentrations of TC $(10 \mathrm{mg} / \mathrm{kg}$ and $30 \mathrm{mg} / \mathrm{kg})$ compared to $20 \mathrm{mg} / \mathrm{kg}$ TC. However, the survival area was higher in the group receiving $10 \mathrm{mg} / \mathrm{kg} \mathrm{TC}$ than in the control group. The TC concentration of $30 \mathrm{mg} / \mathrm{kg}$ had no effect on random flap survival because there was no significant difference between the $30 \mathrm{mg} / \mathrm{kg}$ TC group and the control group.

Many factors contribute to flap survival, especially perfusion of the distal partial flap. ${ }^{19,23}$ Numerous researchers have performed many techniques to improve flap perfusion, including microsurgery techniques, such as surgical delay, ${ }^{4,24}$ venous supercharge, or artery supercharge, ${ }^{25-27}$ as well as medicinal chemistry, such as vascular endothelium growth factors. ${ }^{7}$ However, the disadvantages of the above microsurgery techniques are 
obvious, such as prolonged operative time, ${ }^{28}$ which may highly increase the risk of postoperative complications regardless of the success or failure of the operation. In addition, although growth factor drugs have been proven efficient in improving flap survival, ${ }^{29}$ their high prices do not make them popular in clinical settings. Therefore, in this study, we studied TC, which is reasonably priced, and showed that it has good therapeutic effects.

Trans-cinnamaldehyde, eugenol, and linalool represent $82.5 \%$ of the total composition of Cinnamomum zeylanicum (CZ). ${ }^{12}$ Trans-cinnamaldehyde accounts for approximately $49.9-62.8 \%$ of the total amount of bark oil. ${ }^{30,31}$ To accurately investigate the role of TC in flap survival, TC with a purity of $\geq 95 \%$ was used in this study. The literature has reported that through in vitro and in vivo studies of cinnamaldehyde in animals and humans, CZ has many beneficial effects on health, such as anti-inflammatory properties, antimicrobial activity, reducing cardiovascular disease, boosting cognitive function, and reducing the risk of colonic cancer. ${ }^{31}$ However, there is no report of TC affecting flap survival, let alone its optimum concentration, despite its efficacy. In this study, we found that TC efficiently improved random flap survival and that $20 \mathrm{mg} / \mathrm{kg}$ TC was the optimum concentration. Ayano et al, ${ }^{16}$ reported that cinnamaldehyde induces relaxation of rat aortae and that this vasodilation effect is significantly inhibited by $\mathrm{N}^{\mathrm{G}}$-nitro-L-arginine methyl ester (L-NAME, a nonselective nitric oxide synthase inhibitor). Therefore, we speculated that TC may induce relaxation of subdermal vessels through the nitric oxide synthase pathway to improve flap perfusion. Finally, the results of macroscopic flap survival demonstrated that coadministration of L-NAME and the most effective dose $(20 \mathrm{mg} / \mathrm{kg})$ of TC had no significant effect when compared with the control group, revealing the involvement of the nitric oxide synthase pathway.

To exclude the influence of solvents and inhibitors themselves used in this experiment, we used control groups that received only normal saline, DMSO, and L-NAME. The flap survival results showed that there

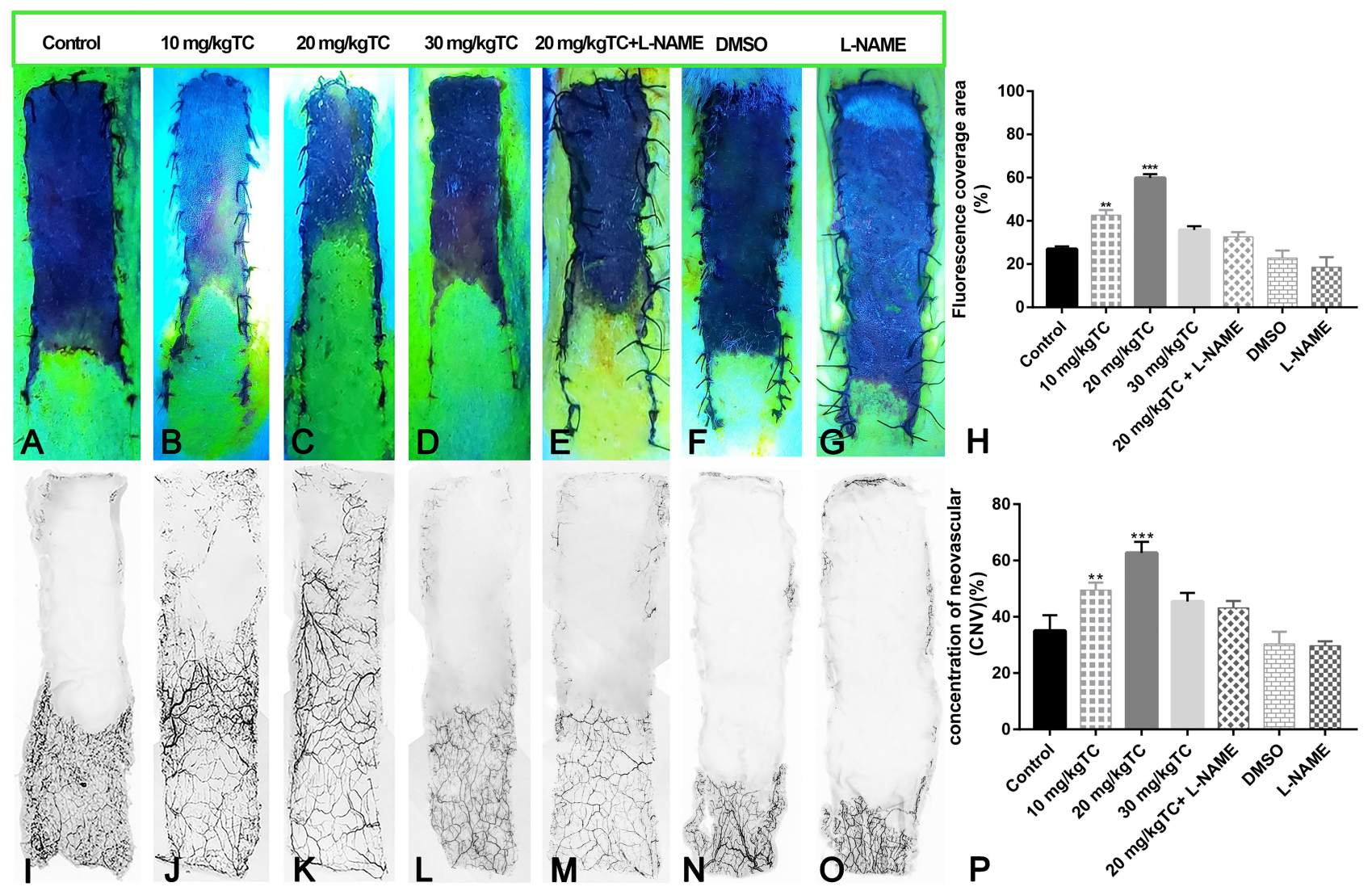

Figure 3 The perfusion area and vessel density of flaps on postoperative day 7 .

Notes: $(\mathbf{A}-\mathbf{G})$ Fluorescein staining of the seven groups on postoperative day 7. (H) Statistical results of flap fluorescein staining. (I-O) Images of the arteriography with oxide-gelatine contrast medium of the seven groups. (P) Statistical percentage of flap microvascular density. Significant differences between groups are indicated as $* * * p<$ 0.001 , **p $<0.01$. 
Table 2 Percentage of Fluorescence Coverage and Concentration of Neovascular (CNV)

\begin{tabular}{|c|c|c|c|}
\hline Groups & Treatment Identification & Fluorescence Coverage Rates (Mean \pm SD\%) & CNV (Mean \pm SD\%) \\
\hline 1 & Control ( $0.9 \%$ normal saline $)$ & $27 \pm 10$ & $38 \pm 5$ \\
\hline 2 & $10 \mathrm{mg} / \mathrm{kg}$ of $\mathrm{TC}$ & $41 \pm 8$ & $54 \pm 8$ \\
\hline 3 & $20 \mathrm{mg} / \mathrm{kg}$ of TC & $58 \pm 12$ & $72 \pm 10$ \\
\hline 4 & $30 \mathrm{mg} / \mathrm{kg}$ of TC & $24 \pm 9$ & $42 \pm 8$ \\
\hline 5 & $20 \mathrm{mg} / \mathrm{kg}$ of TC + L-NAME & $26 \pm 11$ & $40 \pm 7$ \\
\hline 6 & I mL/kg DMSO (0.5\%) & $21 \pm 6$ & $29 \pm 4$ \\
\hline 7 & L-NAME & $18 \pm 8$ & $28 \pm 3$ \\
\hline
\end{tabular}

Abbreviations: TC, trans-cinnamaldehyde; L-NAME, $\mathrm{N}^{\mathrm{G}}$-nitro-L-arginine methyl ester; DMSO, dimethylsulfoxide.

was no significant difference among the above control groups $(\mathrm{P}>0.05)$, indicating that the solvents and inhibitors used in our study did not influence the experiment. The results of laser Doppler showed that TC increased flap perfusion and that $20 \mathrm{mg} / \mathrm{kg}$ TC showed the highest flap perfusion among all groups. We speculated that this may be the direct reason for improving flap survival. Arteriography with oxide-gelatine contrast medium is a traditional and acknowledged technique to measure vascular density. ${ }^{19,32}$ The results of arteriography with oxidegelatine contrast medium and fluorescent staining revealed the tendency of neovascular formation in groups receiving $10 \mathrm{mg} / \mathrm{kg}$ and $20 \mathrm{mg} / \mathrm{kg}$ TC. However, the neovascular percentage in the $20 \mathrm{mg} / \mathrm{kg}$ TC group was much higher than that in the $10 \mathrm{mg} / \mathrm{kg} \mathrm{TC}$ group, indicating that TC also contributes to neovascular formation. Based on the above results, we selected sections from the normal saline control group, $10 \mathrm{mg} / \mathrm{kg}$ TC group, $20 \mathrm{mg} / \mathrm{kg}$ TC group, $20 \mathrm{mg} / \mathrm{kg}$ TC group + L-NAME and L-NAME to evaluate histopathological staining (H\&E and Masson's trichrome) and immunohistochemistry (IHC). H\&E staining, which is a frequently used for measuring vascular density, ${ }^{33}$
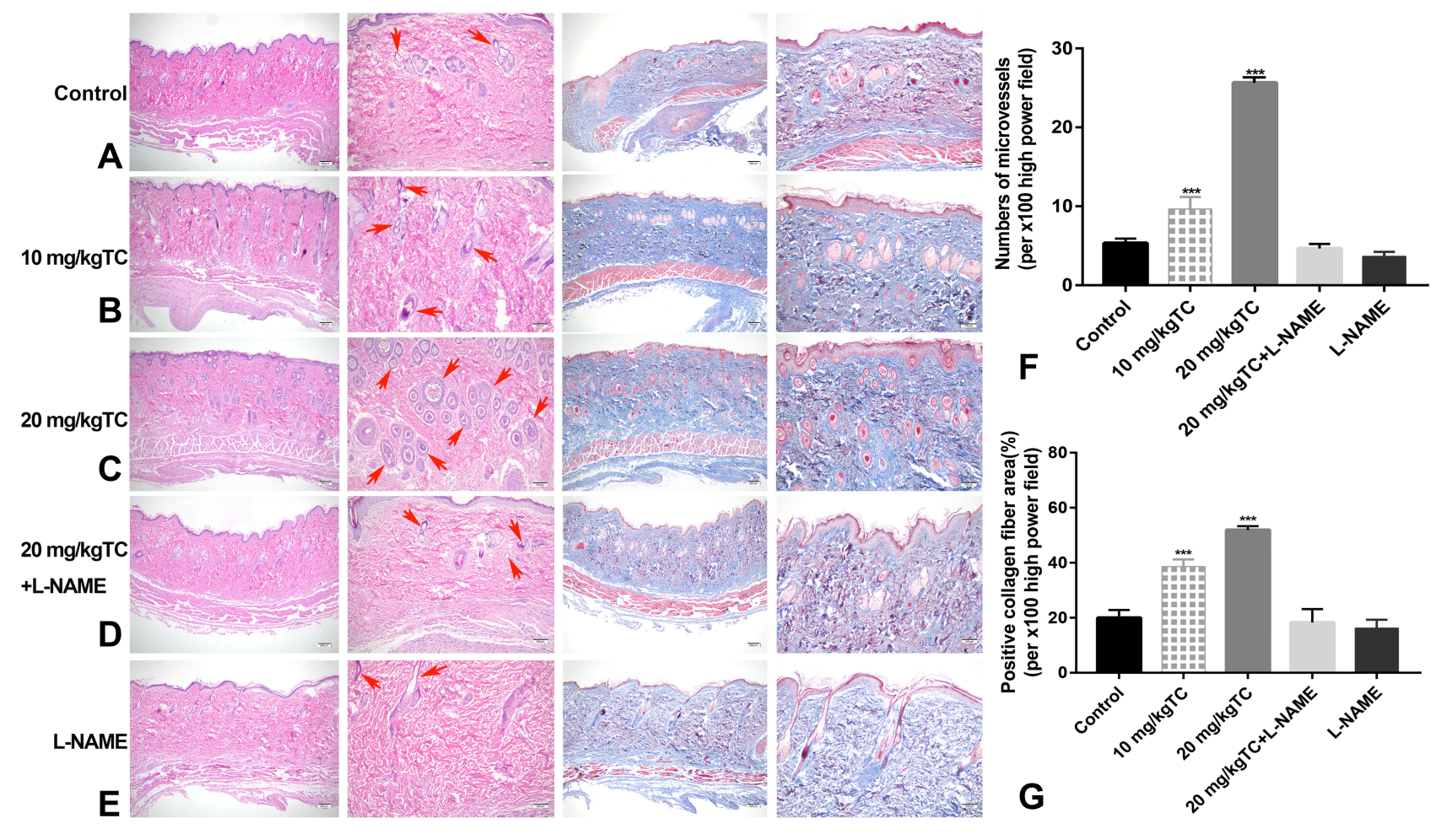

Figure 4 Histopathological staining on postoperative day 7.

Notes: (A-E, Left) H\&E staining of the five groups receiving normal saline, $10 \mathrm{mg} / \mathrm{kg} \mathrm{TC}, 20 \mathrm{mg} / \mathrm{kg}$ TC, $20 \mathrm{mg} / \mathrm{kg}$ TC+L-NAME and L-NAME. Microvessels are indicated by red arrows. (A-E, Right) Masson's trichrome staining of the five groups receiving normal saline, $10 \mathrm{mg} / \mathrm{kg}$ TC, $20 \mathrm{mg} / \mathrm{kg}$ TC, $20 \mathrm{mg} / \mathrm{kg}$ TC+L-NAME and L-NAME. (F) Statistical results of vessel count using H\&E staining. (G) Statistical percentage of fibrin deposition using Masson's trichrome staining. Significant differences between groups are indicated as $* * * * 0.001$.

Abbreviations: H\&E, haematoxylin and eosin; TC, trans-cinnamaldehyde; L-NAME, $N^{G}$-nitro-L-arginine methyl ester. 
confirmed that $20 \mathrm{mg} / \mathrm{kg}$ TC contributed to higher vascular density than $10 \mathrm{mg} / \mathrm{kg} \mathrm{TC}$. In addition, skin regeneration is regarded as a complex process involving inflammation, proliferation, epithelialization, angiogenesis, remodelling, and scarring. ${ }^{34}$ Masson's trichrome staining was used in this study to detect fibrin deposition in flaps. ${ }^{19}$ Comparing fibrosis among the groups revealed a significantly higher level of fibrosis in the $20 \mathrm{mg} / \mathrm{kg}$ TC group than in the $10 \mathrm{mg} / \mathrm{kg}$ TC group, thereby indicating that $20 \mathrm{mg} / \mathrm{kg}$ TC effectively contributes to flap survival via fibrin deposition.

CD34 is a common molecular biological marker of microvascular density (MVD). ${ }^{35}$ The results of H\&E and IHC of CD34 consistently revealed that the $20 \mathrm{mg} / \mathrm{kg}$ TC group had the most intensive microvessels among the five groups, suggesting that $20 \mathrm{mg} / \mathrm{kg} \mathrm{TC}$ is beneficial to microangiogenesis. It has been reported that endothelial cells can sense shear stress and perform signal conversion to upregulate the expression of endothelial nitric oxide synthase (eNOS) to expand blood vessels. ${ }^{36}$ Thus, we speculate that vessels in the $20 \mathrm{mg} / \mathrm{kg}$ TC group will be expanded due to high expression of eNOS. The results of IHC of eNOS showed the highest level in the $20 \mathrm{mg} / \mathrm{kg}$ TC group among the five groups. This phenomenon was verified through laser Doppler perfusion images that showed dilation of proximal flap vessels with high blood perfusion in the $20 \mathrm{mg} / \mathrm{kg}$ TC group. Vascular endothelial growth factors (VEGFs) are regarded as crucial factors for

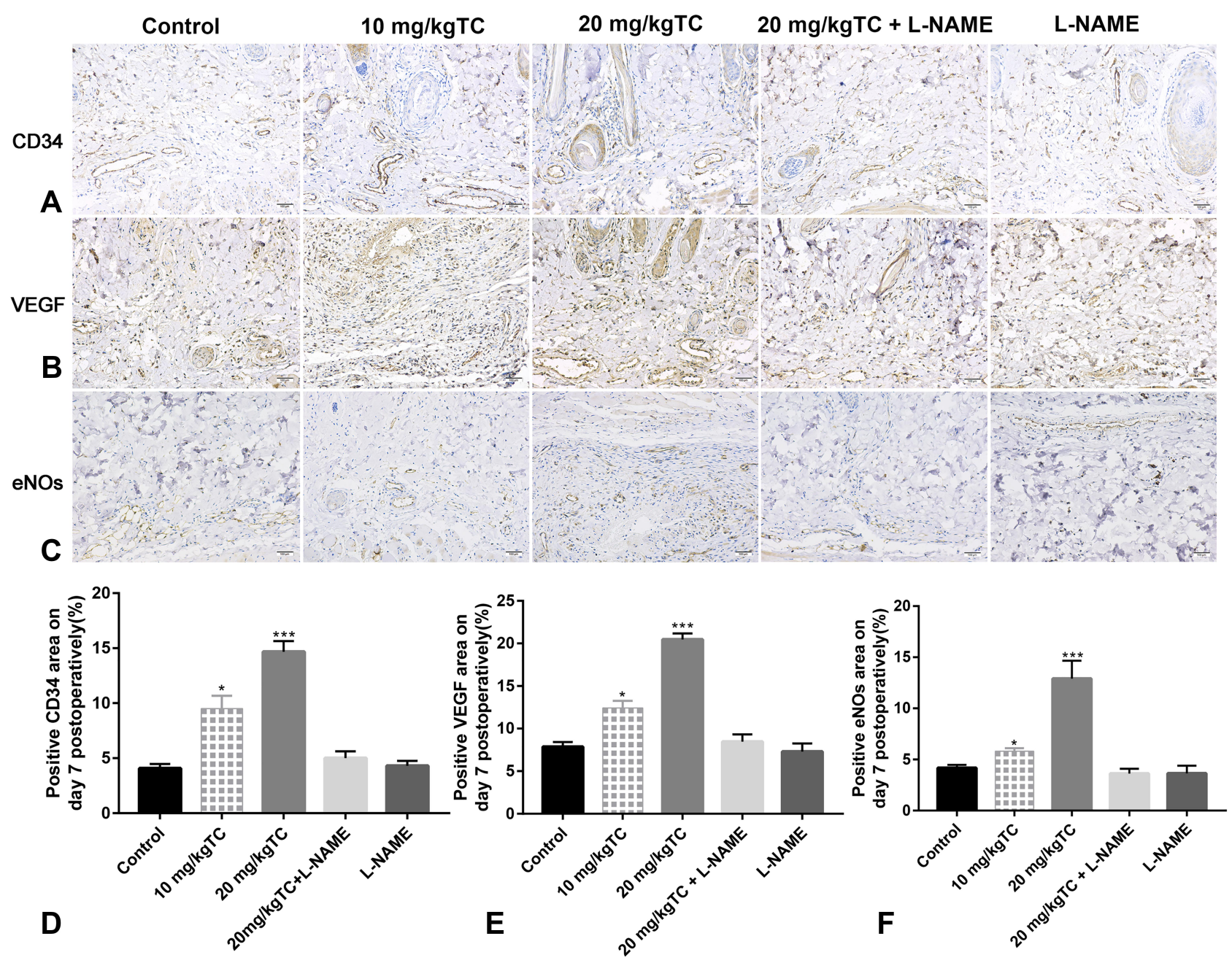

Figure 5 Immunohistochemistry staining on postoperative day 7.

Notes: (A) IHC staining of CD34 in the five groups receiving normal saline, $10 \mathrm{mg} / \mathrm{kg} \mathrm{TC}, 20 \mathrm{mg} / \mathrm{kg} \mathrm{TC}, 20 \mathrm{mg} / \mathrm{kg}$ TC+L-NAME and L-NAME. (B) IHC staining of eNOS in the five groups receiving normal saline, $10 \mathrm{mg} / \mathrm{kg} \mathrm{TC}, 20 \mathrm{mg} / \mathrm{kg} \mathrm{TC}, 20 \mathrm{mg} / \mathrm{kg} \mathrm{TC}+\mathrm{L}-\mathrm{NAME}$ and L-NAME. (C) IHC staining of VEGF in the five groups receiving normal saline, $10 \mathrm{mg} / \mathrm{kg}$ TC, $20 \mathrm{mg} / \mathrm{kg}$, TC20 mg/kg TC+L-NAME and L-NAME. (D) Statistical percentage of CD34-positive area. (E) Statistical percentage of eNOS-positive area. (F) Statistical percentage of VEGF-positive area. Significant differences between groups are indicated as $*^{* *} p<0.001, *_{p}<0.05$.

Abbreviations: IHC, immunohistochemistry; TC, trans-cinnamaldehyde; L-NAME, $N^{G}$-nitro-L-arginine methyl ester; eNOS, endothelial nitric oxide synthase; VEGF, vascular endothelial growth factor. 
angiogenesis. $^{37}$ The results of IHC of VEGF suggested that the expression of VEGF in the $20 \mathrm{mg} / \mathrm{kg}$ TC group was the highest among the five groups. This phenomenon was also verified through the results of modified angiography with oxide-gelatine, measuring the concentration of microvascular density.

However, the present study still has limitations. First, we did not take other organ samples of rats, in addition to skin flaps, to check TC toxicity in the rat body, although $20 \mathrm{mg} / \mathrm{kg}$ TC was found to be effective in improving random flap survival. Second, the postoperative observation of rats lasted only one week. Further studies are warranted to evaluate the long-term effects of TC on various organs in rats in vivo.

\section{Conclusion}

Trans-cinnamaldehyde increased flap survival by activating the nitric oxide pathway and contributing to angiogenesis. The TC concentration of $20 \mathrm{mg} / \mathrm{kg}$ is recommended after comparing groups administered different doses of TC due to the lower adverse effects and greater safety.

\section{Acknowledgment}

This study was supported by the Wenzhou Municipal Science and Technology Bureau (Grant number: Y20180667), the Zhejiang Traditional Chinese Medicine Administration (Grant number: 2018ZB079), and the Zhejiang Provincial Natural Science Foundation of China (Grant number: LY18H060010).

\section{Disclosure}

The authors report no conflicts of interest in this work.

\section{References}

1. Ichiro Hashimoto YA, Ishida $\mathrm{S}$, Kashiwagi $\mathrm{K}$, Mineda $\mathrm{K}$, Yutaro Yamashita, et al. Development of skin flaps for reconstructive surgery random pattern flap to perforator flap. J Med Invest. 2016;63 (3-4):159-162. doi:10.2152/jmi.63.159

2. McGregor IA, G. M. Axial and random pattern flaps. Br J Plast Surg. 1973;26(3):202-213. doi:10.1016/0007-1226(73)90003-9

3. Taylor GI, Palmer JH. The vascular territories (angiosomes) of the body: experimental study and clinical applications. Br J Plast Surg. 1987;40(113):1987. doi:10.1016/0007-1226(87)90185-8

4. Lineaweaver WC, Lei MP, Mustain W, Oswald TM, Cui D, Zhang F. Vascular endothelium growth factor, surgical delay, and skin flap survival. Ann Surg. 2004;239(6):866-73; discussion 873-5. doi:10.1097/01.sla.0000128682.53915.92

5. Chang H, Minn KW, Imanishi N, Minabe T, Nakajima H. Effect of venous superdrainage on a four-territory skin flap survival in rats. Plast Reconstr Surg. 2007;119(7):2046-2051. doi:10.1097/01. prs.0000260590.44798.9c
6. Chang H, Nobuaki I, Minabe T, Nakajima H. Comparison of three different supercharging procedures in a rat skin flap model. Plast Reconstr Surg. 2004;113(1):277-283. doi:10.1097/01. PRS.0000095953.57320.2F

7. Guo Y, Y. G, Li J, Xiao H. Effects of natural and recombinant hirudin on VEGF expression and random skin flap survival in a venous congested rat model. Int Surg. 2013;98(1):82-87. doi:10.9738/ CC171.1

8. Emery FM, Bomberger RA, D B M. The effect of nifedipine on skin-flap survival. Plast Reconstr Surg. 1990;85(1):61-63. doi:10.1097/00006534-199001000-00011

9. Shalom A, Friedman T, Westreich M. Effect of aspirin and heparin on random skin flap survival in rats. Dermatol Surg. 2008;34(6):785-90; discussion 790. doi:10.1111/j.1524-4725.2008.34147.x

10. Kim HJ, Xu L, Chang KC, et al. Anti-inflammatory effects of anthocyanins from black soybean seed coat on the keratinocytes and ischemia-reperfusion injury in rat skin flaps. Microsurgery. 2012;32(7):563-570. doi:10.1002/micr.22019

11. Garcia-Pedraza JA, Garcia M, Martin ML, Moran A. Pharmacological evidence that 5-HT1D activation induces renal vasodilation by NO pathway in rats. Clin Exp Pharmacol Physiol. 2015;42(6):640-647. doi:10.1111/1440-1681.12397

12. Priyanga Ranasinghe SP, Sirimal Premakumara GA, Galappaththy P, Constantine GR, Katulanda P. Medicinal properties of 'true' cinnamon (Cinnamomum zeylanicum): a systematic review. $B M C$ Complement Altern Med. 2013;22(13):275. doi:10.1186/1472-688213-275

13. Farag MR, Alagawany M, Tufarelli V. In vitro antioxidant activities of resveratrol, cinnamaldehyde and their synergistic effect against cyadox-induced cytotoxicity in rabbit erythrocytes. Drug Chem Toxicol. 2017;40(2):196-205. doi:10.1080/01480545.2016.1193866

14. Kim ME, Na JY, Lee JS. Anti-inflammatory effects of trans-cinnamaldehyde on lipopolysaccharide-stimulated macrophage activation via MAPKs pathway regulation. Immunopharmacol Immunotoxicol. 2018;40(3):219-224. doi:10.1080/ 08923973.2018.1424902

15. Subash Babu P, Prabuseenivasan S, Ignacimuthu S. Cinnamaldehyde-a potential antidiabetic agent. Phytomedicine. 2007;14(1):15-22. doi:10.1016/j.phymed.2006.11.005

16. Ayano Yanaga, Takako Nakagawa, Hiroaki Hikiami, Naotoshi Shibahara, Yutaka Shimada. Cinnamaldehyde induces endothelium-dependent and -independent vasorelaxant action on isolated rat aorta. Biol Pharm Bull. 2006;29(12):2415-2418. doi:10.1248/bpb.29.2415

17. Hansen-Schwartz J, Lovland Hoel N, Nilsson E, Tfelt-Hansen P, Edvinsson L. Endothelium-dependent relaxant responses to selective 5 -HT (1B/1D) receptor agonists in the isolated middle cerebral artery of the rat. J Vasc Res. 2003;40(6):561-566. doi:10.1159/000075806

18. Chin LC, Kumar P, Palmer JA, et al. The influence of nitric oxide synthase 2 on cutaneous wound angiogenesis. $\mathrm{Br} J$ Dermatol. 2011;165(6):1223-1235. doi:10.1111/j.1365-2133.2011.10599.x

19. Luo X, Liu J, Chen H, et al. The feasibility and survival mechanism of a large free flap supported by a novel hybrid perfusion mode. Oral Oncol. 2019;101:104506. doi:10.1016/j.oraloncology.2019.104506

20. Tao Y, Hu S, Lui KW, Chen S, Tang M, Chen S. Quantitative regression analysis of the cutaneous vascular territories in a rat model. Surgical Radiologic Anatomy. 2011;33(9):789-799. doi:10.1007/s00276-011-0809-7

21. Rabiolo A, Bignami F, Rama P, Ferrari G, Vessel J. A new tool for semiautomatic measurement of corneal neovascularization. Investigative Ophthalmol Visual Sci. 2015;56(13):8199. doi:10.1167/iovs.15-17098

22. Shu J, Dolman GE, Duan J, Qiu G, Ilyas M. Statistical colour models: an automated digital image analysis method for quantification of histological biomarkers. Biomed Eng Online. 2016;15(1). doi:10.1186/s12938-016-0161-6 
23. David F, B AW, Makki FM, Trites JR, Mark taylor S, Hart RD. Late free flap failure in head and neck reconstruction: a systematic review. Ear Nose Throat J. 2018;97(7):213-216. doi:10.1177/014556131809700712

24. Richard J, James R, Khalique Z, Jerome Z, Janice A. TRAM Flap perforator ligation and the delay phenomenon: development of an endoscopic/laparoscopic delay procedure. Plast Reconstr Surg. 1998;101(6):1503-1511. doi:10.1097/00006534-199805000-00012

25. Suh YC, Ahn MR, Kim SY, Kim YJ, Lee JH. Superficial inferior epigastric artery-supercharged deep inferior epigastric artery perforator flap-based breast reconstruction for a thin patient: a case report. Microsurgery. 2019;39(7):651-654. doi:10.1002/micr.30503

26. Fang F, Liu M, Xiao J, Zhuang Y. Arterial supercharging is more beneficial to flap survival due to quadruple dilation of venules. J Surg Res. 2020;247:490-498. doi:10.1016/j.jss.2019.09.056

27. Kim EJ, Lee HJ, Mun GH. Muscle-splitting approach to thoracoacromial vein for superdrainage in deep inferior epigastric artery perforator flap breast reconstruction. Microsurgery. 2019;39 (3):228-233. doi:10.1002/micr.30421

28. Ayestaray B, Yonekura K, Motomura H, Ziade M. A comparative study between deep inferior epigastric artery perforator and thoracoacromial venous supercharged deep inferior epigastric artery perforator flaps. Ann Plast Surg. 2019;39(3):78-82. doi:10.1097/ SAP.0000000000000581

29. Chen GJ, Chen YH, Yang XQ, Li ZJ. Nano-microcapsule basic fibroblast growth factor combined with hypoxia-inducible factor-1 improves random skin flap survival in rats. Mol Med Rep. 2016;13 (2):1661-1666. doi:10.3892/mmr.2015.4699

30. Singh G, Maurya S, deLampasona MP, Catalan CAN. A comparison of chemical, antioxidant and antimicrobial studies of cinnamon leaf and bark volatile oils, oleoresins and their constituents. Food Chem Toxicol. 2007;45(9):1650-1661. doi:10.1016/j.fct.2007.02.031
31. Simic A, Sokovic MD, Ristic M, Grujic-Jovanovic S, Vukojevic J, Marin PD. The chemical composition of some Lauraceae essential oils and their antifungal activities. Phytother Res. 2004;18 (9):713-717. doi:10.1002/ptr.1516

32. Yan H, He Z, Li Z, et al. Large prefabricated skin flaps based on the venous system in rabbits. Plast Reconstr Surg. 2013;132(3):372e380e. doi:10.1097/PRS.0b013e31829acf3d

33. Xi S, Cheng S, Lou J, et al. A preliminary study of the effects of venous drainage position on arterial blood supply and venous return within the conjoined flap. Plast Reconstr Surg. 2019;143(2):322e328e. doi:10.1097/PRS.0000000000005256

34. Sorg H, Tilkorn DJ, Hager S, Hauser J, Mirastschijski U. Skin wound healing: an update on the current knowledge and concepts. Eur Surg Res. 2017;58(1-2):81-94. doi:10.1159/000454919

35. Ivanovic Z, Kwon S-M, Lee J-H, et al. Cross talk with hematopoietic cells regulates the endothelial progenitor cell differentiation of CD34 positive cells. PLoS One. 2014;9(8).

36. Kimiko Yamamoto HI, Ando J. Shear stress augments mitochondrial ATP generation that triggers ATP release and $\mathrm{Ca} 2+6$ signaling in vascular endothelial cells. Am J Physiol Heart Circ Physiol. 2018;315 (5):H1477-H1485. doi:10.1152/ajpheart.00204.2018

37. Millauer B, Wizigmann-Voos S, Schnurch H, et al. High affinity VEGF binding and developmental expression suggest Flk-1 as a major regulator of vasculogenesis and angiogenesis. Cell. 1993;72:835-846. doi:10.1016/0092-8674(93)90573-9

\section{Publish your work in this journal}

Drug Design, Development and Therapy is an international, peerreviewed open-access journal that spans the spectrum of drug design and development through to clinical applications. Clinical outcomes, patient safety, and programs for the development and effective, safe, and sustained use of medicines are a feature of the journal, which has also been accepted for indexing on PubMed Central. The manuscript management system is completely online and includes a very quick and fair peer-review system, which is all easy to use. Visit http://www. dovepress.com/testimonials.php to read real quotes from published authors. 